\title{
AQUEOUS TWO-PHASE SYSTEMS: SIMPLE ONE-STEP PROCESS FORMULATION AND PHASE DIAGRAM FOR CHARACTERISATION
}

\author{
Florence Dumas $^{1}$, Emilie Roger ${ }^{1}$, Johanna Rodriguez ${ }^{1}$, Lazhar Benyahia ${ }^{2}$, Jean-Pierre Benoit ${ }^{1}$ \\ ${ }^{1}$ Micro et Nanomedecines Translationnelles, MINT, UNIV Angers, UMR INSERM 1066, UMR CNRS \\ 6021, Angers, France \\ ${ }^{2}$ Institut des Molécules et Matériaux du Mans, IMMM, UNIV Le Mans, UMR 6283 CNRS, Le Mans, France
}

Published in Colloid and Polymer Science (DOI: 10.1007/s00396-020-04748-8)

\begin{abstract}
Aqueous two-phase system (ATPS), also called "water-in-water" emulsion, is a multicomponent system (aqueous droplets dispersed in a continuous aqueous phase), formed after gently mixing two aqueous solutions of immiscible polymers. It allows the formation of different compartments without adding any organic solvent, according to a green process. Nevertheless, the kinetic stability of ATPS is generally difficult to control. Phase diagrams, which express the concentration of a polymer as a function of that of the other in solution, are used to characterise ATPS and to determine conditions for preparing emulsions from selected polymers. In this study, dextran and polyethylene oxide aqueous mixtures with different processing parameters are investigated. Phase diagrams are generated through two automatic methods (Turbiscan and LUMiSizer ${ }^{\circledR}$ technologies) and a manual one. With the diagrams obtained, it is concluded that the purity of polyethylene oxide affects the ATPS, whereas the use of polymers in powder or in solution form has no effect. In view of this result, it is allowed to prepare ATPS formulation by a simple one-step process from polymers in powder form.
\end{abstract}

Keywords: Aqueous two-phase system, Phase diagram, Dextran, Polyethylene oxide

\section{Introduction}

Aqueous two-phase systems (ATPS), also known as "water-in-water" emulsions, are mixtures of hydrosoluble but incompatible polymers in water. In these systems, droplets (in general in the micrometer range) of an aqueous solution of one of the polymers are dispersed in a continuous phase consisting of an aqueous solution of the other polymer ${ }^{1}$. Such systems can be scaled up easily and they are cheap to formulate ${ }^{2}$. Given the mild conditions used and the rapidity of the process ${ }^{3,4}$, ATPS are particularly useful for the separation and purification of biomolecules 5,6 , such as proteins, enzymes (e.g. fungal laccases used in the production of antibiotics, anti-cancer or anaesthetic molecules), monoclonal antibodies (e.g. IgG, anti-CD34) ${ }^{7}$ and even cells ${ }^{8}$ (platelets isolated from blood ${ }^{9}$, confinement of cells to form 3D culture of cancer cells (spheroids) ${ }^{10}$ ). Some differences between the three-dimensional structures of closely related proteins can also be identified by exploring their partitioning in ATPS with different ionic compositions ${ }^{11}$. In medicine, ATPS are used in gene therapy (for the isolation of DNA or nucleic acids) and vaccination (purification and concentration 
of viruses) ${ }^{7}$. ATPS are formed by a "green" process without adding any organic solvent 12,13. This is a particularly attractive characteristic for the agrofood sector, for the detection or extraction of low-molecular weight molecules and residues of medicinal products in water, even at very low concentrations (e.g., residues of antibiotics, pesticides or herbicides) ${ }^{7}$. Various other applications have been developed for ATPS, including uses in the separation of metals or metal ions, textile dyes in washing water, and the extraction of products of fermentation by micro-organisms ${ }^{7}$.

ATPS can be formulated by mixing at least two thermodynamically incompatible polymers in aqueous solution ${ }^{14}$. Aqueous phases including a polymer and a salt (e.g. PEG/phosphate), respectively, can also form ATPS 2. Other systems use different compounds, such as guar gum, hydroxypropyl starch, polysorbate, ethylene or propylene oxide copolymers (EOPO) ${ }^{2}$. Many of the most frequently studied ATPS are based on pairs of dextran and polyethylene oxide polymers of different molecular weights (polyethylene glycol (PEG) or polyethylene oxide (PEO)). These two different types of polymers are immiscible in certain proportions, in which their mixture results in ATPS ${ }^{15}$. PEG polymers, with the formula $\left(-\mathrm{CH}_{2} \mathrm{CH}_{2} \mathrm{O}-\right) \mathrm{n}$, are linear polyethers composed of ethylene glycol monomers. Their chain length is variable, making it possible to adjust their molecular weight for diverse possibilities. A PEG is often referred as "PEO" if its molecular weight exceeds $20 \mathrm{~kg} \cdot \mathrm{mol}^{-1}$. Dextran is a natural polysaccharide polymer produced by bacteria. The use of dextran/PEG polymer combinations to create ATPS presents several advantages for the separation of biomolecules, such as proteins 16. However, such emulsions are restricted by their dynamic behaviour, which leads to droplet deformation ${ }^{14}$. The Quality-byDesign approach ensures high-quality formulation through the use of a welldesigned process. It has already been used to improve our understanding of novel formulation processes, for drug-loaded nanoparticles for example ${ }^{17}$. In this context, many characterisation studies are required during the development of ATPS. Emulsion structure has been shown to depend on viscosity, interfacial tensions, resistance to bending and interfacial permeability ${ }^{18}$. All these properties affect the phase separation process ${ }^{18}$. Many characterisation methods are based on the study of the interfacial tension between the dispersed and continuous phases 14,19-21. Theories and models have been developed to explain the deformation/relaxation behaviour of ATPS 1,22 . The main problem with such formulations is the strong destabilisation displayed by emulsions, in which rapid droplet deformation causes significant instability. Some studies 19,20 have characterised emulsions with phase diagrams 23 , to determine the optimal formulation conditions. Phase diagrams express the concentration of a polymer as function of that of another polymer in solution (Fig.1). They present "tie-lines", which connect two points corresponding to the concentrations of the totally separated phases. They also show the binodal curve, which defines the limits of the multiphase system. Below this curve, the polymers are miscible. Phase diagrams can be used to evaluate the effects of temperature and polymer molecular weight on the composition of the aqueous phases of emulsions, for example. At higher polymer molecular weights, the binodal curves are pushed down to lower polymer concentrations ${ }^{24}$. These diagrams can be used to determine the conditions required for the formation of emulsions from given polymers as function as their concentrations. For biomedical applications, ATPS containing less than $5 \mathrm{wt} \%$ of either PEG (25 to $45 \mathrm{~kg} \cdot \mathrm{mol}^{-1}$ ) and dextran (450 to 650 $\mathrm{kg} . \mathrm{mol}^{-1}$ ) are more suitable due to short dissolution time and low interfacial tension 25. However, ATPS composed of PEG $\left(8 \mathrm{~kg} \cdot \mathrm{mol}^{-1}\right)$ and dextran $\left(500 \mathrm{~kg} \cdot \mathrm{mol}^{-1}\right.$ or $66.9 \mathrm{~kg} \cdot \mathrm{mol}^{-1}$ ), with concentrations of $9.6 \mathrm{wt}$ $\%$ of PEG and 1.0 wt \% of dextran (500 $\left.\mathrm{kg} . \mathrm{mol}^{-1}\right)$, or concentrations of $4.2 \mathrm{wt} \%$ of 
PEG and $9.8 \mathrm{wt} \%$ of dextran $\left(66.9 \mathrm{~kg} \cdot \mathrm{mol}^{-}\right.$ $\left.{ }^{1}\right)$ provided the best conditions for lipase or lutein extraction, respectively ${ }^{26,27}$. Finally, for the couple of PEO (200 kg. $\left.\mathrm{mol}^{-1}\right)$ and dextran (500 kg.mol $\left.{ }^{-1}\right)$, Balakrishnan, et al. determined phase diagram studying concentrations up to $8 \mathrm{wt} \%$ of PEO and 4 $\mathrm{wt} \%$ of dextran ${ }^{19}$. Thus, in this study, the phase diagrams of PEO (molecular weight $200 \mathrm{~kg} \cdot \mathrm{mol}^{-1}$ ) and dextran (molecular weight $500 \mathrm{~kg} \cdot \mathrm{mol}^{-1}$ ) mixtures as a means of characterising ATPS were explored in this last range of concentration. A workability area was identified for this pair of polymers, and a binodal curve and phase diagram were generated. One manual and two automatic methods of characterisation (Turbiscan and LUMiSizer $^{\circledR}$ technologies) were compared. LUMiSizer ${ }^{\circledR}$ uses cutting-edge STEP- (space and time-resolved extinction profiles) technology whereas Turbiscan TOWER uses S-MLS (static multiple light scattering). Both these technologies involve optical methods for the characterisation of concentrated liquid dispersions without the need for dilution. Near-infrared light source extinction profiles are obtained from transmission detectors that recover signals by scanning from the bottom to the top of the sample. The main difference between these two technologies is the destabilisation process, which is accelerated by centrifugal force in the LUMiSizer $^{\circledR}$ technique. The impacts of polymer state (solid or solution) and PEO purity on the phase diagrams were evaluated.

\section{Material \& Method \\ Materials}

Dextran from Leuconostoc mesenteroides (Mw: $500 \mathrm{~kg} \cdot \mathrm{mol}^{-1}$ ), and polyethylene oxide (PEO) (Mw: $200 \mathrm{~kg} \cdot \mathrm{mol}^{-}$ ${ }^{1}$ ) were provided by Sigma-Aldrich (SaintQuentin Fallavier, France). Water purified with the MilliQ filtration system (Millipore, Paris, France) was used for all formulations.

\section{Methods}

\section{PEO purification process}

Impurities present in the PEO were removed by dissolving the $\mathrm{PEO}$ in water and centrifuging (5810R centrifuge, Eppendorf AG Hamburg, Germany) for 5 minutes at 4,000 rpm. The impurities were removed as the pellet. The supernatant was centrifuged several times under the same conditions until no impurities pellet was obtained. The supernatant containing PEO was then freezedried (Lyovac GT2 (Steris, Germany)/CC505 (Huber, Germany)) to obtain a purified PEO powder.

\section{Preparation of aqueous mixtures of dextran and PEO}

For mixtures of polymers in solutions (liquid state), aqueous stock solutions of 100 $\mathrm{mg} / \mathrm{mL}$ dextran and $200 \mathrm{mg} / \mathrm{mL}$ PEO were first prepared. These two solutions were then mixed in different proportions.

For mixtures of polymers in powder form (solid state), the dextran and PEO powders were weighed directly and dissolved in water.

\section{Binodal determination}

The binodal curve was determined by the visual method. Different concentrations of PEO and dextran mixtures (ranging from $0.0 \mathrm{wt} \%$ to 2.0 and $4.0 \mathrm{wt} \%$, respectively) were studied, with the polymers in powder form or in aqueous solution. The binodal points were determined by visual for signs of phase separation (interface observation) after three days at room temperature. If phase separation occurred, two phases were observed, corresponding to an emulsion. If no phase separation occurred, there was only a single phase, corresponding to a solution. Obtained results were reported on a map constructed with initial concentration of each polymer. Thus, a first global workability map was designed based on formulations from polymers in powder form with non-purified PEO. The edges of the binodal curve were then studied under different conditions (PEO purified and non-purified, and polymers in solution or in powder form).

\section{Phase diagram generation}

Phase diagrams were obtained by mixing polymers to obtain final concentrations of $2.0 \mathrm{wt} \%$ dextran and 1.0 to 
$8.0 \mathrm{wt} \%$ PEO. The effects of the initial state of the polymers (solution or powder) and PEO purity were studied. Phase separation was investigated with one manual and two automatic methods.

Manual method: aqueous mixtures were stored at room temperature for 5 days and the height of each phase was measured manually.

Turbiscan automatic method: in a 4 $\mathrm{mL}$ cylindrical cell, aqueous mixtures were stored at room temperature for 5 days and analysed with a Turbiscan TOWER (Formulaction, Toulouse, France). Analyses were performed for 4 hours ( 1 scan every 5 minutes) at $25^{\circ} \mathrm{C}$ and the height of each phase was measured graphically with Turbisoft software. Further details of this method are provided in the Supporting Information (Fig. S3).

LUMiSizer ${ }^{\circledR}$ automatic method: in a rectangular cell (10 $\mathrm{mm}$-diameter round top), aqueous mixtures were centrifuged in a LUMiSizer $^{\circledR} \quad$ (LUM GmbH, Berlin, Germany) at 4,000 rpm for 5 minutes at room temperature. The two forming phases were clear and the height of each phase was measured graphically with SEPView ${ }^{\circledR}$ software.

An abacus was set up with water in the glassware used to correlate the height and volume of a phase. From obtained equation, the volume of total aqueous mixture (V total) and the volume of each phase (V polymer phase) were determined and the mass of water in the polymer-rich phase ( $\mathrm{m}$ water phase) were calculated according to Eq. 1, assuming a homogeneous distribution of water in the samples. Then, in each polymerrich phase, experimental polymer concentrations were calculated according to Eq. 2.

(1) m water phase (g)

$=\frac{\mathrm{m} \text { water total }(\mathrm{g}) \times \mathrm{V} \text { polymer phase }(\mathrm{mL})}{\mathrm{V} \text { wotal }(\mathrm{mL})}$ $\mathrm{V}$ total $(\mathrm{mL})$

(2) Experimental polymer concentration ( $\mathrm{wt} \%)$ $=\frac{\mathrm{m} \text { polymer }(\mathrm{g})}{\mathrm{m} \text { polymer }(\mathrm{g})+\mathrm{m} \text { water phase }(\mathrm{g})}$ (where $\mathrm{m}$ polymer and $\mathrm{m}$ water total are the initial mass $(\mathrm{g})$ of polymer and water in the total aqueous mixture).

Finally, phase diagrams were generated with the experimental polymer concentrations, and tie-lines were then normalised by bringing the slopes down to theoretical initial concentrations.

\section{Results \& Discussion Binodal}

The binodal, separating the solution from the emulsion of an aqueous mixture of dextran and PEO, is shown Fig. 2. In the studied area, from a dextran concentration of $2.0 \mathrm{wt} \%$, the experimental binodal was found below $2.0 \mathrm{wt} \%$ of PEO. For aqueous mixtures containing $2.0 \mathrm{wt} \%$ dextran (corresponding to the experimental dextran concentration for subsequent phase diagram construction), the experimental binodal was located above 2.0 $\mathrm{wt} \%$ of PEO. Similar results were found for the studied areas described in the Supporting Information (Table S1), whatever the conditions tested: purified and unpurified PEO, and polymers in solution or powder form. The degree of purity of the PEO and the physical state of the polymers therefore had no impact on the binodal for the concentrations tested. However, the binodal obtained here was higher than that reported in the literature ${ }^{19}$. Indeed, at $2.0 \mathrm{wt} \%$ dextran, the binodal curve was determined for a PEO concentration of between $1.75 \mathrm{wt} \%$ and 2.0 wt $\%$ (Fig. 2), whereas Balakrishnan, et al. ${ }^{19}$ obtained the binodal for a PEO concentration below $1.0 \mathrm{wt} \%$. This highlights the margin of error imputed to operator variability for the manual method.

\section{Phase diagram}

Table 1 shows the equations and linear correlation coefficients obtained with the water abacus for each method. These equations were used to calculate the polymer concentration in each phase to define the tieline slopes and to construct the phase diagram. However, a relative error must be imputed to the calculation due to the approximation of polymer density, which is 
significantly different from water density, especially for high concentrations. Similarly, Amrhein, et al. also highlighted the impact of neglecting density in phase diagram construction ${ }^{28}$. For each method, phase diagrams were generated for PEO (from 1.0 to $8.0 \mathrm{wt} \%$ ) and dextran (at $2.0 \mathrm{wt} \%$ ).

Experimental results used to determine tie-lines were presented Table S2. Based on these experimental results, the phase diagrams were normalized to fit theoretical polymer concentrations. Phase diagrams were compared by superimposition and the interpretation of the results was based on differences in tie-line slopes at the same polymer concentrations. For all the conditions tested, at $2.0 \mathrm{wt} \%$ dextran, the aqueous phase system had a single phase with $1.0 \mathrm{wt} \%$ and $1.5 \mathrm{wt} \%$ PEO.

Indeed, according to the experimental binodal line obtained, polymer concentrations were too low for the formation of an emulsion. Below the binodal line, the studied system formed a stable homogeneous mixture ${ }^{29}$. Whatever the experimental conditions, for $2.0 \mathrm{wt} \%$ PEO, two phases were observed after emulsion destabilisation. Weight percent values are indicated in Table S2, but tie-lines are not presented here. For points for which polymer concentrations were close to the binodal, the tie-lines crossed the tie-lines of high-concentration points. This pattern of behaviour has been reported before, particularly for polymer-salt ATPS, and can be explained by changes in phase density with compound concentration ${ }^{30}$. Moreover, the measurement at this point was not considered accurate because the refractive indices for light scattering changed on passage through the glassware and the meniscus at the interface between the two phases. At this point, very close to the binodal concentration, there was probably also a commingling of polymers, with incomplete phase separation, even if not visible by eye. Ferreira, et al. described the binodal in terms of saturation or space occupation by polymers ${ }^{29}$. The impact of the physical state of the polymer (solid or solution) was then evaluated by superimposing phase diagrams for the different methods used. The phase diagrams obtained are presented in Fig. 3 and Fig. 4 for experimental concentrations of 2.0 $\mathrm{wt} \%$ dextran and $1.0 \mathrm{wt} \%$ to $8.0 \mathrm{wt} \% \mathrm{PEO}$, to focus on the emulsion area. These concentrations were chosen because they are already studied in the literature from polymers in solution ${ }^{19}$. Results with unpurified PEO were presented in Supporting Information (Fig. S1). The phase diagram in Fig. 3A was established with the manual method (method used for binodal determination) and shows the results obtained for polymers in powder or solution form. Following the superimposition of tielines for the $2.0 \mathrm{wt} \%$ dextran points, no difference in the PEO concentration in the PEO-rich phase was observed with polymers in either powder or solution form and whatever the method used (Fig. 3A, Fig. 3B and Fig. 3C). For the manual method, the dextran-rich phase seemed to be more concentrated in ATPS formed from polymers in powder form than in those formed from polymers in solution (Fig. 3A). However, automatic methods identified no differences in the dextran-rich phases (Fig. 3B and Fig. 3C). The manual method appeared less accurate for determining phase heights, and consequently volumes. In Fig. 4A, showing phase diagrams established with the manual method, differences were observed when tielines for $2.0 \mathrm{wt} \%$ dextran were superimposed. However, as discussed above, the inaccuracy of the manual method made it impossible to draw any firm conclusions about these results. The analyses performed with the automatic methods revealed no effect of PEO purity on PEO concentration in the PEO-rich phase (Fig. 4B and Fig. 4C). By contrast, dextran concentration tended to increase in the dextran-rich phase after PEO purification (Fig. 4B and Fig. 4C). Impurities in PEO raw material was identified in the literature as silica particles ${ }^{19}$.

Silica impurities present in the emulsion can sediment out during the 
separation process, as silica has a higher density (approximately 2 g. $\mathrm{cm}^{-3}$ ) than PEO and dextran ones (close to 1 g.cm ${ }^{-3}{ }^{31}$. The resulting error on the final concentrations in each phase led to imprecision in the diagram. These findings are consistent with those of a previous study highlighting the utility of automatic methods for the accurate characterisation of ATPS ${ }^{28}$. Thus, the greater reliability of automatic methods results in an absence of difference between phase diagrams generated with powders and phase diagrams generated with solutions. Thus, these results demonstrate that, both powder and solution forms of polymers can be used indiscriminately to prepare ATPS in these range of polymers concentration. The impact of polymer purity for polymers in powder form was also investigated. Phase diagrams for the various methods with and without purification of PEO were superimposed (Fig. 4) (Results for polymers in solution were reported in Supporting Information (Fig. S2)). Silica would therefore have been found in the bottom dextran phase, resulting in an overestimation of the height and volume of the dextran-rich phase and a lower estimation of concentration than for purified PEO. The concentration of PEO in the PEO-rich phase would probably be unaffected in the phase diagram, because adding low-solubility compounds such as silica would modify the total volume of the ATPS, the apparent volume of bottom phase (the dextran-rich phase), but not that of the top phase (PEOrich phase). The degree of polymer purity therefore had an impact on the phase diagram. For all conditions (purified and unpurified PEO, polymers in solution or in powder form) the information provided by phase diagrams differed with the method used. Finally, a comparison of the manual method with the Turbiscan and LUMiSizer ${ }^{\circledR}$ methods showed that the automatic methods were more reliable for ATPS characterisation. Optimisation of the accuracy of phase diagram construction required complete phase separation. In the system studied, phase separation occurred at low polymer concentrations because of large sizes of the molecules ${ }^{32}$. Interactions with water increased with the molecular size of the polymers. However, this configuration, with a continuous top phase (continuous phase located on top after separation, as for PEO in this case), was not optimal for phase separation. Indeed, it has already been shown that partitioning is faster for a continuous bottom phase ${ }^{33}$. The application of a centrifugal force to accelerate phase separation, as with the LUMiSizer ${ }^{\circledR}$ method, may be useful for overcoming viscosity or molecular size parameters, which affect the duration of phase separation. In any case, the centrifugal force parameters must be optimised for the system, to prevent potential losses of the product of interest ${ }^{34}$. The use of gravitational force for partitioning, as in the Turbiscan method, is also potentially useful, for phase separation in mild conditions. However, this method is more timeconsuming. These two automatic methods provided equivalent results. The choice of equipment should therefore be based on the desired application.

\section{Conclusion}

Many applications for ATPS have been described. However, understanding phase separation behaviour remains a challenge that must be resolved before this formulation process can be scaled up according to a Quality by Design approach.

ATPS prepared with dextran and polyethylene oxide were characterised through phase diagrams obtained by three different methods. A workability area was defined by determining the binodal and tielines composing the phase diagram for this pair of polymers. The physical state (in solution or solid) of the polymers had no effect on the phase diagram. The impact of polymer (PEO) purity was also investigated and $\mathrm{PEO}$ purification was found to result in a higher concentration of dextran in the dextran-rich phase, highlighting the importance of using high-purity raw materials.

In conclusion, automatic methods were more accurate, faster and reliable than the manual 
method for characterising ATPS. Due to approximations in height determinations from the profiles obtained, assays remain the most accurate method of phase diagram construction. Nevertheless, automatic methods, such as the Turbiscan and LUMiSizer $^{\circledR}$ methods, are very easy to use and to quickly generate phase diagrams. So they have a great interest for the comparison of different experimental conditions and for scale up processes. In this study, the results obtained with these methods highlighted the importance of the degree of purity of the

\section{REFERENCES}

1 L. M. C. Sagis, Dynamics of encapsulation and controlled release systems based on water-in-water emulsions: Liposomes and polymersomes, Phys. A Stat. Mech. its Appl., 2009, 388, 2579-2587.

2 F. Ruiz-Ruiz, J. Benavides, O. Aguilar and M. Rito-Palomares, Aqueous twophase affinity partitioning systems: Current applications and trends, $J$. Chromatogr. A, 2012, 1244, 1-13.

3 W. . G. Fan CE., Charged protein partitioning in aqueous polyethylene glycol-dextran two-phase systems: salt effects., Sep. Sci. Technol., 1999, 34, 423-438.

4 W. Fan, U. Bakir and C. E. Glatz, Contribution of protein charge to partitioning in aqueous two-phase systems, Biotechnol. Bioeng., 1998, 59, 461-470.

5 J. Jiang and J. M. Prausnitz, Molecular Thermodynamics for Partitioning of Native and Denatured Proteins in Aqueous Two-Phase Systems, J. Phys. Chem. B, 2000, 104, 7197-7205.

6 H.-O. Johansson, J. Persson and F. Tjerneld, Thermoseparating water/polymer system: A novel onepolymer aqueous two-phase system for protein purification, Biotechnol. Bioeng., 1999, 66, 247-257.

7 M. Iqbal, Y. Tao, S. Xie, Y. Zhu, D. polymer. Finally, the most attractive results were obtained by comparing polymers forms. Thus, a simple one-step process, easily scalable in industrial process, from a mixture of polymers in powder form with adding water can be proposed to prepare ATPS formulation.

Abbreviations: ATPS: aqueous two-phase system; Dex: dextran; PEO: polyethylene oxide; $\mathrm{Wt} \%$ : mass concentration as a percentage.

Chen, X. Wang, L. Huang, D. Peng, A. Sattar, M. A. B. Shabbir, H. I. Hussain, S. Ahmed and Z. Yuan, Aqueous twophase system (ATPS): an overview and advances in its applications, Biol. Proced. Online, 2016, 18, 18.

8 E. Atefi, R. Joshi and H. Tavana, Effect of Molecular Weight of Phase Polymers on Partition of Cells in Aqueous TwoPhase Systems, MRS Adv., 2017, 2, 2415-2426.

9 E. Sumida, Y. Iwasaki, K. Akiyoshi and S. Kasugai, Platelet Separation From Whole Blood in an Aqueous Two-Phase System With Water-Soluble Polymers, J. Pharmacol. Sci., 2006, 101, 91-97.

10 E. Atefi, S. Lemmo, D. Fyffe, G. D. Luker and H. Tavana, High throughput, polymeric aqueous two-phase printing of tumor spheroids, Adv. Funct. Mater., 2014, 24, 6509-6515.

11 A. Zaslavsky, P. Madeira, L. Breydo, V. N. Uversky, A. Chait and B. Zaslavsky, High throughput characterization of structural differences between closely related proteins in solution, Biochim. Biophys. Acta - Proteins Proteomics, 2013, 1834, 583-592.

12 D. M. A. Buzza, P. D. I. Fletcher, T. K. Georgiou and N. Ghasdian, Water-inwater emulsions based on incompatible polymers and stabilized by triblock copolymers-templated polymersomes, 
Langmuir, 2013, 29, 14804-14814.

13 R. J. H. Stenekes, O. Franssen, E. M. G. van Bommel, D. J. A. Crommelin and W. E. Hennink, The use of aqueous PEG/dextran phase separation for the preparation of dextran microspheres, Int. J. Pharm., 1999, 183, 29-32.

14 L. M. C. Sagis, Dynamics of controlled release systems based on water-in-water emulsions: A general theory, J. Control. Release, 2008, 131, 5-13.

15 S. Ghosh, R. Vijayalakshmi and T. Swaminathan, Evaluation of an alternative source of dextran as a phase forming polymer for aqueous two-phase extractive system, Biochem. Eng. J., 2004, 21, 241-252.

16 M. W. Edelman, E. Van Der Linden and R. H. Tromp, Phase separation of aqueous mixtures of poly(ethylene oxide) and dextran, Macromolecules, 2003, 36, 7783-7790.

17 D. Leng, K. Thanki, E. Fattal, C. Foged and $M$. Yang, Engineering of budesonide-loaded lipid-polymer hybrid nanoparticles using a quality-bydesign approach, Int. J. Pharm., 2018, 548, 740-746.

18 E. Scholten, L. M. C. Sagis and E. van der Linden, Effect of bending rigidity and interfacial permeability on the dynamical behavior of water-in-water emulsions, J. Phys. Chem. B, 2006, 110, 3250-3256.

19 G. Balakrishnan, T. Nicolai, L. Benyahia and D. Durand, Particles trapped at the droplet interface in waterin-water emulsions, Langmuir, 2012, 28, 5921-5926.

20 B. T. Nguyen, T. Nicolai and L. Benyahia, Stabilization of water-inwater emulsions by addition of protein particles, Langmuir, 2013, 29, 1065810664.

21 E. Atefi, J. A. Mann and H. Tavana, Ultralow Interfacial Tensions of Aqueous Two-Phase Systems Measured Using Drop Shape, Langmuir, 2014, 30, 9691-9699.

22 L. M. C. Sagis, Dynamics of
Encapsulation and Controlled Release Systems Based on Water-in-Water Emulsions: Negligible Surface Rheology, J. Phys. Chem. B, 2008, 112, 13503-13508.

23 A. Kaul, The Phase Diagram, Methods Biotechnol., 2000, 11, 11-21.

24 D. Forciniti, C. K. Hall and M.-R. Kula, Influence of polymer molecular weight and temperature on phase composition in aqueous two-phase systems, Fluid Phase Equilib., 1991, 61, 243-262.

25 H. Shin, C. Han, J. M. Labuz, J. Kim, J. Kim, S. Cho, Y. S. Gho, S. Takayama and J. Park, High-yield isolation of extracellular vesicles using aqueous two-phase system, Sci. Rep., 2015, 5, 13103.

26 C. W. Ooi, S. L. Hii, S. M. M. Kamal, A. Ariff and T. C. Ling, Extractive fermentation using aqueous two-phase systems for integrated production and purification of extracellular lipase derived from Burkholderia pseudomallei, Process Biochem., 2011, 46, 68-73.

27 A. Chavez-Santoscoy, J. Benavides, W. Vermaas and M. Rito-Palomares, Application of Aqueous Two-Phase Systems for the Potential Extractive Fermentation of Cyanobacterial Products, Chem. Eng. Technol., 2010, 33, 177-182.

28 S. Amrhein, M. L. Schwab, M. Hoffmann and J. Hubbuch, Characterization of aqueous two phase systems by combining lab-on-a-chip technology with robotic liquid handling stations, J. Chromatogr. A, 2014, 1367, 68-77.

29 L. A. Ferreira, V. N. Uversky and B. Y. Zaslavsky, Modified binodal model describes phase separation in aqueous two-phase systems in terms of the effects of phase-forming components on the solvent features of water, $J$. Chromatogr. A, 2018, 1567, 226-232.

30 W. Rao, Y. Wang, J. Han, L. Wang, T. Chen, Y. Liu and L. Ni, Cloud Point and Liquid-Liquid Equilibrium Behavior of 
Thermosensitive Polymer L61 and Salt Aqueous Two-Phase System, J. Phys. Chem. B, 2015, 119, 8201-8208.

31 L. Fele and M. Fermeglia, Partition Coefficients of Proteins in Poly(ethylene glycol) + Dextran + Water at $298 \mathrm{~K}$, J. Chem. Eng. Data, 1996, 41, 331-334.

32 R. Hatti-Kaul, Aqueous Two-Phase Systems: A General Overview, Mol. Biotechnol., 2001, 19, 269-278.

33 J. A. Asenjo and B. A. Andrews,
Aqueous two-phase systems for protein separation: Phase separation and applications, J. Chromatogr. A, 2012, 1238, 1-10.

34 M. A. Torres-Acosta, K. MayoloDeloisa, J. González-Valdez and M. Rito-Palomares, Aqueous Two-Phase Systems at Large Scale: Challenges and Opportunities, Biotechnol. J., 2018, 1800117, 1800117. 


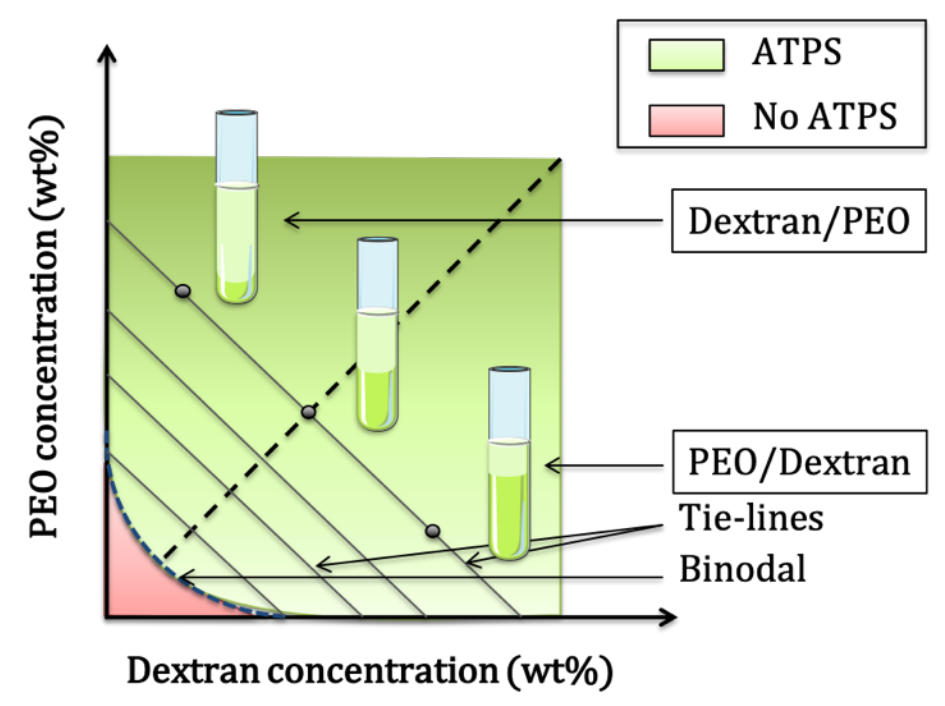

Figure 1. Schematic representation of phase diagram with binodal curve separating ATPS from no ATPS region, and tie-lines obtained for the couple of polymers PEO and dextran

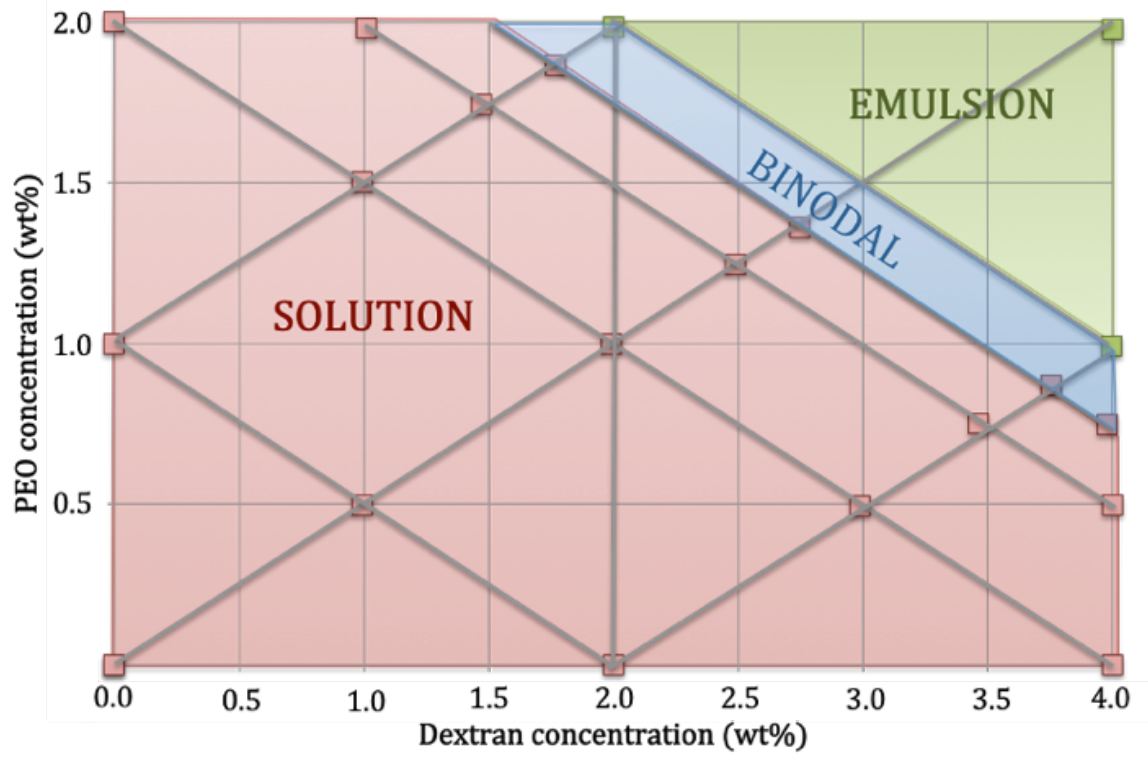

Figure 2. Map representing the binodal (blue area) for the aqueous dextran and PEO system separating the one-phase region (solution, red area) from the two-phase region (emulsion, green area). Aqueous systems were prepared from PEO (unpurified) and dextran in powder form, with concentrations ranging from $0.0 \mathrm{wt} \%$ to 2.0 and $4.0 \mathrm{wt} \%$, respectively. 


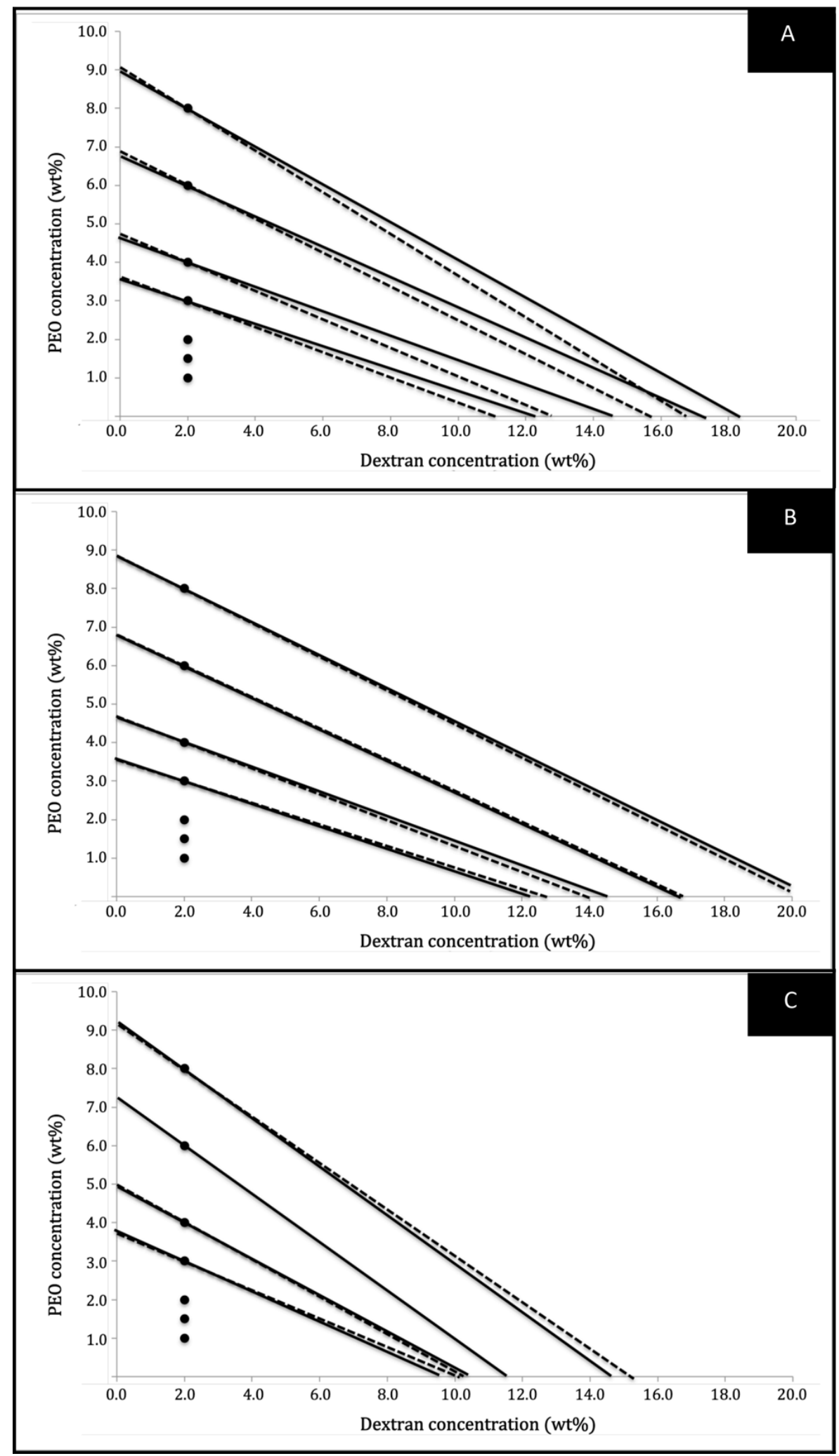

Figure 3. Normalised phase diagrams obtained with purified PEO from polymers in powder (solid line) and solution (dotted line) form, with three different methods: A - Manual method, B - Turbiscan, C - LUMiSizer ${ }^{\circledR}$ 


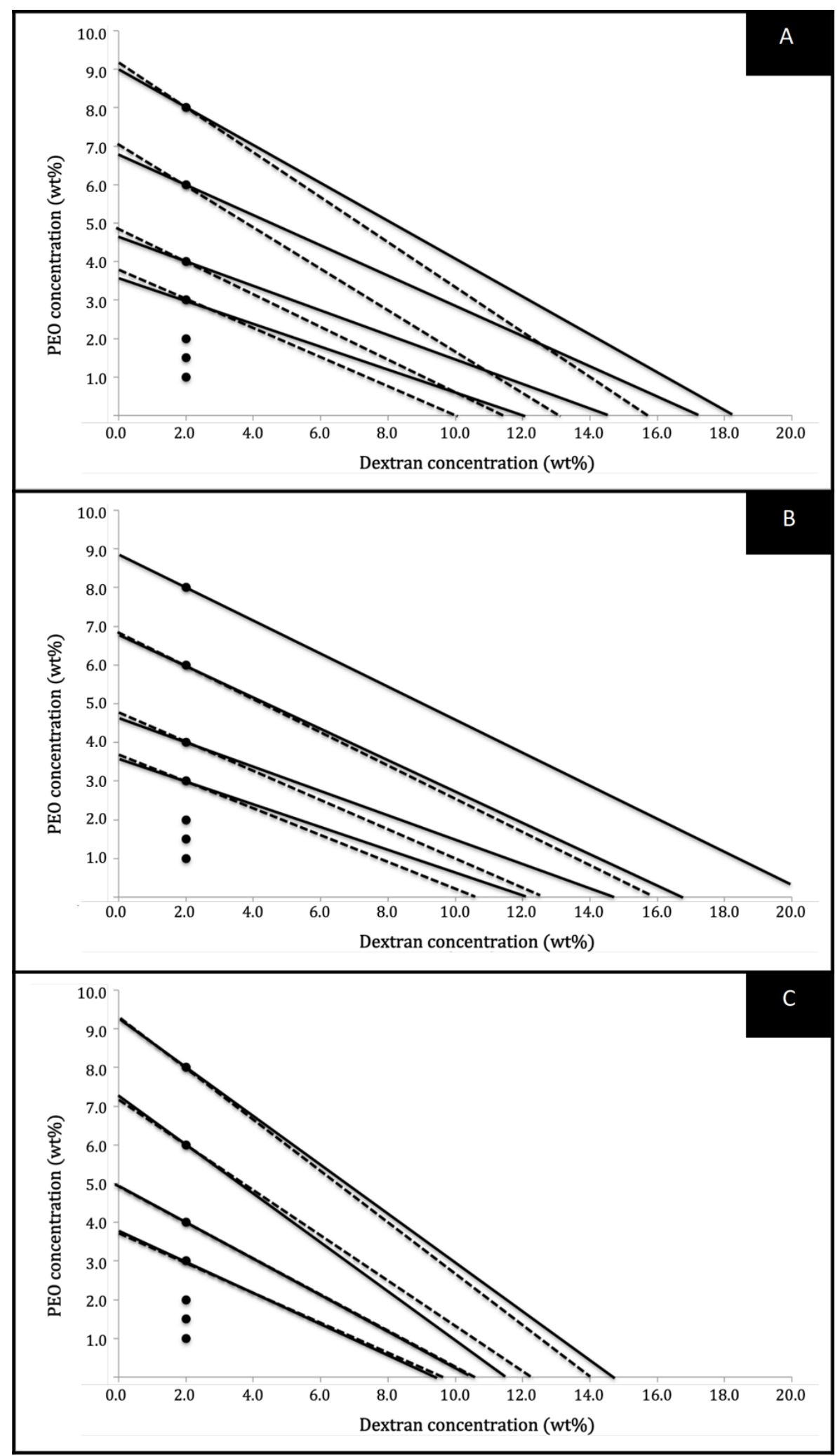

Figure 4. Normalised phase diagrams obtained with polymers in powder form, with purified (solid line) and unpurified (dotted line) PEO, for the three different methods tested: A - Manual method, B - Turbiscan, C - LUMiSizer ${ }^{\circledR}$ 
Table 1. Equations and correlation coefficients for the abacus for the manual and automatic methods, with $\mathrm{Y}$ representing the volume of water (in $\mathrm{mL}$ ) and $\mathrm{X}$ the height of the phase (in $\mathrm{mm})$ in the vial used

Method

Manual and Turbiscan

Equation

Correlation coefficient
$\mathrm{Y}=0.1336 \mathrm{X}$

$\mathrm{R}^{2}=0.9990$
LUMiSizer $^{\circledR}$

$\mathrm{Y}=0.0682 \mathrm{X}$

$\mathrm{R}^{2}=0.9998$ 


\section{Supporting Information}

Table S1. Results obtained for binodal determination, by comparing polymers in powder and solution form, and purified and unpurified PEO. Concentrations ranged from 1 to $2 \mathrm{wt} \%$ for dextran and 2 to $4 \mathrm{wt} \%$ for PEO

\begin{tabular}{|c|c|c|c|c|c|c|c|c|c|}
\hline \multirow{3}{*}{$\begin{array}{c}\text { Polymer form } \\
\text { Purity } \\
\text { Concentration }(w t \%)\end{array}$} & \multicolumn{4}{|c|}{ Powder } & \multicolumn{4}{|c|}{ Solution } & \multirow{3}{*}{ Results } \\
\hline & \multicolumn{2}{|c|}{ Purified PEO } & \multicolumn{2}{|c|}{ Unpurified PEO } & \multicolumn{2}{|c|}{ Purified PEO } & \multicolumn{2}{|c|}{ Unpurified PEO } & \\
\hline & PEO & Dextran & PEO & Dextran & PEO & Dextran & PEO & Dextran & \\
\hline \multirow{9}{*}{ Experimental data } & $1.0 \%$ & $1.9 \%$ & $1.0 \%$ & $2.1 \%$ & $1.2 \%$ & $2.0 \%$ & $1.1 \%$ & $2.0 \%$ & Solution \\
\hline & $1.5 \%$ & $2.0 \%$ & $1.5 \%$ & $2.0 \%$ & $1.7 \%$ & $2.0 \%$ & $1.5 \%$ & $1.8 \%$ & Solution \\
\hline & $2.1 \%$ & $2.0 \%$ & $2.0 \%$ & $2.0 \%$ & $2.2 \%$ & $2.0 \%$ & $2.2 \%$ & $2.0 \%$ & Emulsion \\
\hline & $1.0 \%$ & $3.0 \%$ & $1.0 \%$ & $3.0 \%$ & $0.9 \%$ & $3.2 \%$ & $1.2 \%$ & $3.0 \%$ & Solution \\
\hline & $1.5 \%$ & $3.0 \%$ & $1.5 \%$ & $3.0 \%$ & $1.5 \%$ & $3.1 \%$ & $1.9 \%$ & $3.1 \%$ & Emulsion \\
\hline & $2.1 \%$ & $3.0 \%$ & $2.0 \%$ & $3.0 \%$ & $2.1 \%$ & $3.1 \%$ & $2.4 \%$ & $3.1 \%$ & Emulsion \\
\hline & $1.0 \%$ & $4.0 \%$ & $1.0 \%$ & $4.0 \%$ & $1.1 \%$ & $4.1 \%$ & $1.2 \%$ & $4.2 \%$ & Emulsion \\
\hline & $1.5 \%$ & $4.0 \%$ & $1.6 \%$ & $4.0 \%$ & $1.4 \%$ & $4.3 \%$ & $1.8 \%$ & $4.1 \%$ & Emulsion \\
\hline & $2.1 \%$ & $4.0 \%$ & $2.0 \%$ & $4.0 \%$ & $2.0 \%$ & $4.3 \%$ & $2.3 \%$ & $4.2 \%$ & Emulsion \\
\hline
\end{tabular}


Table S2. Polymer concentrations (\%wt) determined under different conditions of purity (purified and unpurified PEO) and polymer state (solution or powder), with the manual method and two automatic methods: Turbiscan and LUMiSizer ${ }^{\circledR}$

\begin{tabular}{|c|c|c|c|c|c|c|c|c|c|c|c|c|c|c|c|}
\hline & & & \multicolumn{4}{|c|}{ Manual } & \multicolumn{4}{|c|}{ Turbiscan } & \multicolumn{4}{|c|}{ LUMiSizer ${ }^{\circledR}$} \\
\hline & & \multicolumn{2}{|c|}{$\begin{array}{c}\text { Theoretical } \\
\text { Concentrations } \\
(\%)\end{array}$} & \multicolumn{4}{|c|}{$\begin{array}{c}\text { Experimental } \\
\text { Concentrations } \\
(\%)\end{array}$} & \multicolumn{4}{|c|}{$\begin{array}{c}\text { Experimental } \\
\text { Concentrations } \\
(\%)\end{array}$} & \multicolumn{4}{|c|}{$\begin{array}{c}\text { Experimental } \\
\text { Concentrations } \\
(\%)\end{array}$} \\
\hline E & $\stackrel{B}{E}$ & $\begin{array}{c}\text { Dex } \\
\text { initial }\end{array}$ & $\begin{array}{c}\text { PEO } \\
\text { initia } \\
\quad 1\end{array}$ & $\begin{array}{c}\text { Dex } \\
\text { initial }\end{array}$ & $\begin{array}{c}\text { PEO } \\
\text { initial }\end{array}$ & $\begin{array}{l}\text { Dex in } \\
\text { Dex- } \\
\text { rich } \\
\text { phase }\end{array}$ & $\begin{array}{l}\text { PEO } \\
\text { in } \\
\text { PEO- } \\
\text { rich } \\
\text { phase }\end{array}$ & $\begin{array}{c}\text { Dex } \\
\text { initial }\end{array}$ & $\begin{array}{c}\text { PEO } \\
\text { initial }\end{array}$ & $\begin{array}{l}\text { Dex in } \\
\text { Dex- } \\
\text { rich } \\
\text { phase }\end{array}$ & $\begin{array}{l}\text { PEO } \\
\text { in } \\
\text { PEO- } \\
\text { rich } \\
\text { phase }\end{array}$ & $\begin{array}{c}\text { Dex } \\
\text { initial }\end{array}$ & $\begin{array}{c}\text { PEO } \\
\text { initial }\end{array}$ & $\begin{array}{l}\text { Dex in } \\
\text { Dex- } \\
\text { rich } \\
\text { phase }\end{array}$ & $\begin{array}{l}\text { PEO } \\
\text { in } \\
\text { PEO- } \\
\text { rich } \\
\text { phase }\end{array}$ \\
\hline \multirow{14}{*}{ : } & \multirow{7}{*}{ 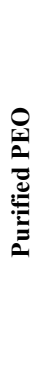 } & 2.0 & 1.0 & 1.9 & 1.0 & N/A & N/A & 1.9 & 1.0 & N/A & N/A & 2.0 & 1.0 & N/A & N/A \\
\hline & & 2.0 & 1.5 & 2.0 & 1.5 & N/A & N/A & 2.0 & 1.5 & N/A & N/A & 2.0 & 1.5 & N/A & N/A \\
\hline & & 2.0 & 2.0 & 2.0 & 2.1 & 12.6 & 2.4 & 2.0 & 2.1 & 13.4 & 2.4 & 2.0 & 2.1 & 10.7 & 2.6 \\
\hline & & 2.0 & 3.0 & 2.0 & 3.1 & 12.7 & 3.6 & 2.0 & 3.1 & 12.2 & 3.7 & 2.0 & 3.0 & 9.5 & 3.8 \\
\hline & & 2.0 & 4.0 & 2.0 & 4.0 & 14.5 & 4.7 & 2.0 & 4.0 & 14.5 & 4.7 & 2.0 & 4.0 & 10.5 & 5.0 \\
\hline & & 2.0 & 6.0 & 2.0 & 6.0 & 17.1 & 6.8 & 2.0 & 6.0 & 16.6 & 6.8 & 2.0 & 6.0 & 11.5 & 7.3 \\
\hline & & 2.0 & 8.0 & 2.0 & 8.1 & 18.6 & 9.2 & 2.0 & 8.1 & 21.0 & 9.0 & 2.0 & 8.0 & 14.8 & 9.3 \\
\hline & \multirow{7}{*}{ 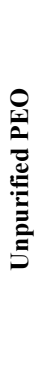 } & 2.0 & 1.0 & 2.0 & 1.0 & N/A & N/A & 2.0 & 1.0 & N/A & N/A & 2.1 & 1.0 & N/A & N/A \\
\hline & & 2.0 & 1.5 & 2.0 & 1.5 & N/A & N/A & 2.0 & 1.5 & N/A & N/A & 2.0 & 1.5 & N/A & N/A \\
\hline & & 2.0 & 2.0 & 2.0 & 2.0 & 10.4 & 2.5 & 2.0 & 2.0 & 11.2 & 2.5 & 2.0 & 2.0 & 10.7 & 2.5 \\
\hline & & 2.0 & 3.0 & 2.0 & 3.0 & 10.0 & 3.8 & 2.0 & 3.0 & 10.5 & 3.7 & 2.0 & 3.0 & 9.4 & 3.8 \\
\hline & & 2.0 & 4.0 & 2.0 & 4.0 & 11.4 & 4.9 & 2.0 & 4.0 & 12.5 & 4.8 & 2.0 & 4.0 & 10.4 & 4.9 \\
\hline & & 2.0 & 6.0 & 2.0 & 6.0 & 13.1 & 7.1 & 2.0 & 6.0 & 15.8 & 6.9 & 2.0 & 6.0 & 12.1 & 7.2 \\
\hline & & 2.0 & 8.0 & 2.0 & 8.0 & 15.5 & 9.2 & 2.0 & 8.0 & N/A & N/A & 2.0 & 8.0 & 13.9 & 9.3 \\
\hline \multirow{14}{*}{$\stackrel{\mathscr{E}}{\stackrel{\varrho}{\Xi}}$} & \multirow{7}{*}{ 管 } & 2.0 & 1.0 & 1.8 & 1.0 & N/A & N/A & 1.8 & 1.0 & N/A & N/A & 1.9 & 0.8 & N/A & N/A \\
\hline & & 2.0 & 1.5 & 1.9 & 1.2 & N/A & N/A & 1.9 & 1.2 & N/A & N/A & 1.9 & 1.4 & N/A & N/A \\
\hline & & 2.0 & 2.0 & 1.8 & 1.8 & 21.2 & 2.0 & 1.8 & 1.8 & 27.7 & 2.0 & 1.9 & 2.0 & 35.8 & 2.1 \\
\hline & & 2.0 & 3.0 & 1.9 & 2.8 & 10.2 & 3.5 & 1.9 & 2.8 & 11.6 & 3.4 & 1.9 & 2.8 & 9.4 & 3.5 \\
\hline & & 2.0 & 4.0 & 1.9 & 3.6 & 11.4 & 4.3 & 1.9 & 3.6 & 12.2 & 4.2 & 2.0 & 3.8 & 9.6 & 4.8 \\
\hline & & 2.0 & 6.0 & 2.0 & 5.4 & 14.1 & 6.3 & 2.0 & 5.4 & 15.1 & 6.2 & 1.9 & 5.9 & 87.6 & 6.0 \\
\hline & & 2.0 & 8.0 & 2.0 & 7.5 & 15.7 & 8.6 & 2.0 & 7.5 & 18.9 & 8.4 & 2.0 & 8.0 & 15.3 & 9.2 \\
\hline & \multirow{7}{*}{ 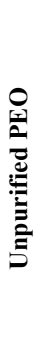 } & 2.0 & 1.0 & 1.9 & 0.9 & N/A & N/A & 1.9 & 0.9 & N/A & N/A & 1.9 & 0.9 & N/A & N/A \\
\hline & & 2.0 & 1.5 & 1.6 & 1.3 & N/A & N/A & 1.6 & 1.3 & N/A & N/A & 1.9 & 1.5 & N/A & N/A \\
\hline & & 2.0 & 2.0 & 1.9 & 1.8 & 16.9 & 2.1 & 1.9 & 1.8 & 16.9 & 2.1 & 1.9 & 2.0 & 57.6 & 2.1 \\
\hline & & 2.0 & 3.0 & 1.9 & 2.7 & 10.6 & 3.3 & 1.9 & 2.7 & 10.4 & 3.4 & 1.9 & 3.1 & 9.2 & 3.9 \\
\hline & & 2.0 & 4.0 & 2.0 & 3.6 & 12.4 & 4.2 & 2.0 & 3.6 & 11.0 & 4.3 & 1.9 & 4.1 & 9.9 & 5.1 \\
\hline & & 2.0 & 6.0 & 1.9 & 5.6 & 17.3 & 6.3 & 1.9 & 5.6 & 14.6 & 6.4 & 2.0 & 6.1 & 12.2 & 7.3 \\
\hline & & 2.0 & 8.0 & 2.0 & 7.5 & 17.2 & 8.4 & 2.0 & 7.5 & 18.5 & 8.4 & 2.1 & 8.3 & 14.8 & 9.7 \\
\hline
\end{tabular}




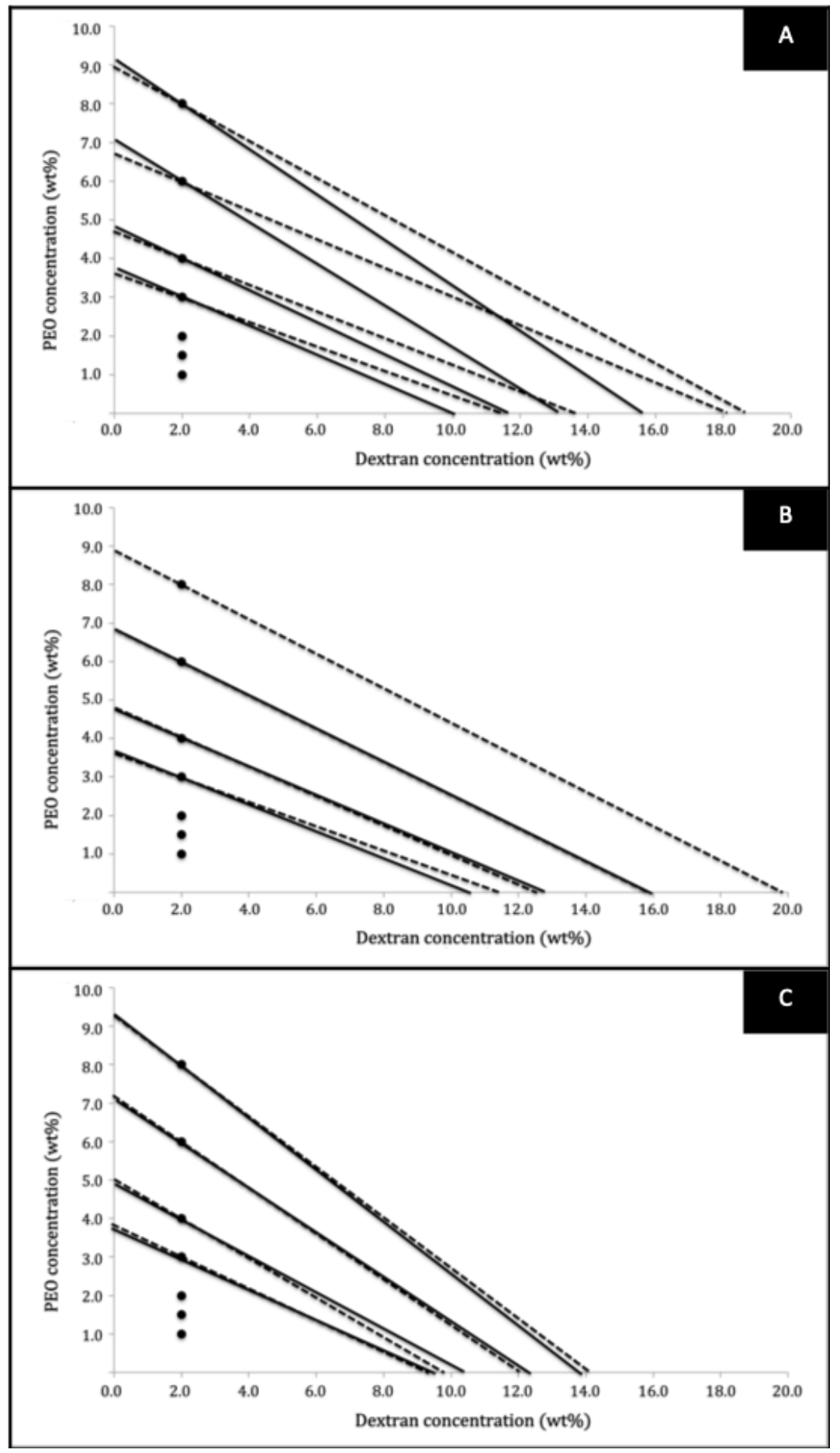

Figure S1. Normalised phase diagrams obtained with unpurified PEO, from polymers in powder form (solid line) and polymers in solution (dotted line), for the three different methods tested: A - Manual method, B - Turbiscan, C - LUMiSizer $\left.{ }^{\circledR}\right)$ - The tie-line at $8.0 \mathrm{wt} \%$ PEO was not determined with the Turbiscan method, due to the impossibility to determine the height of the sample accurately from the transmission profile obtained (B) 


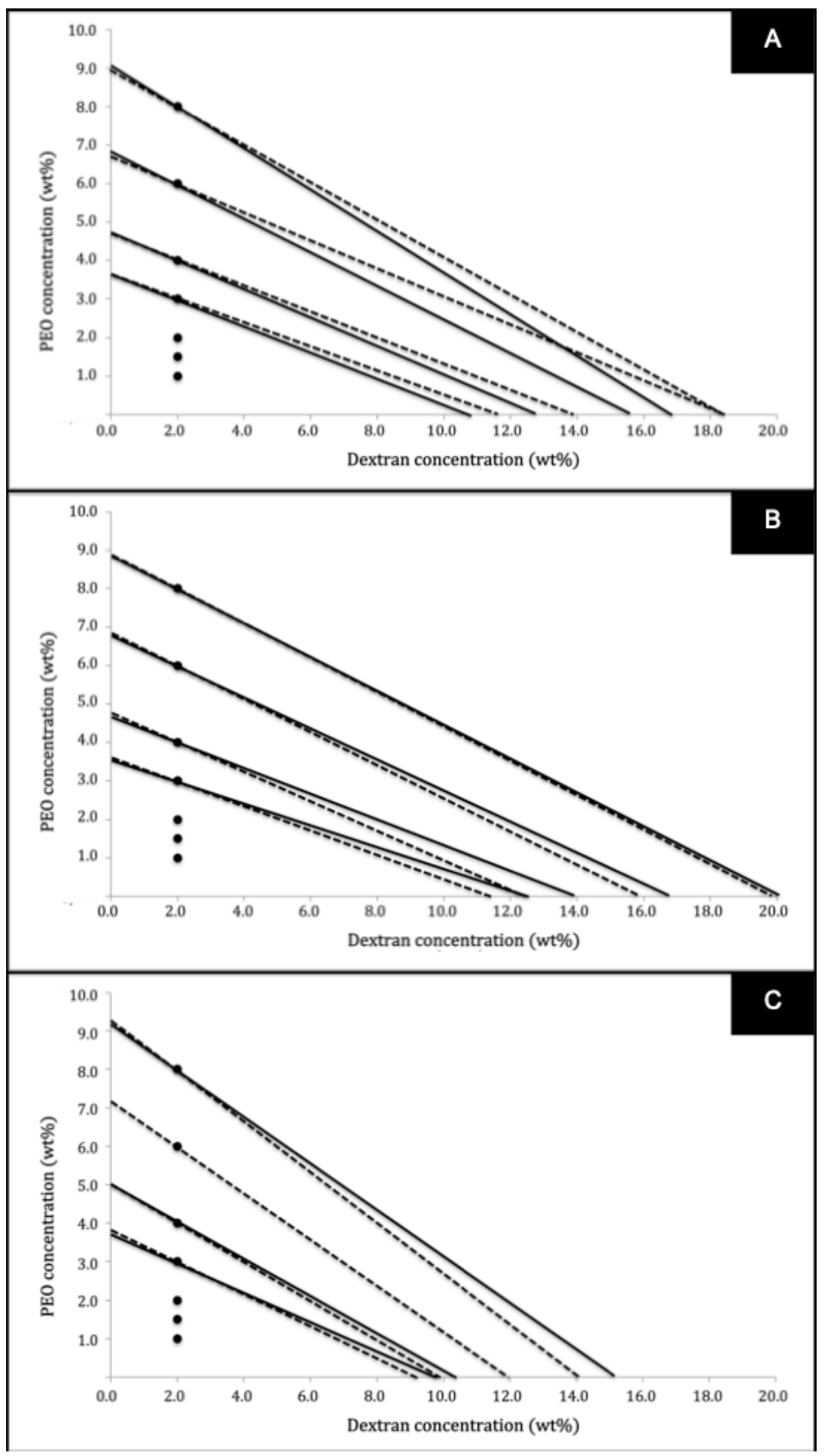

Figure S2. (Normalised phase diagrams obtained with polymers in solution, with purified (solid line) and unpurified (dotted line) PEO, for the three different methods tested: A - Manual method, B - Turbiscan, C - LUMiSizer $\left.{ }^{\circledR}\right)$ 


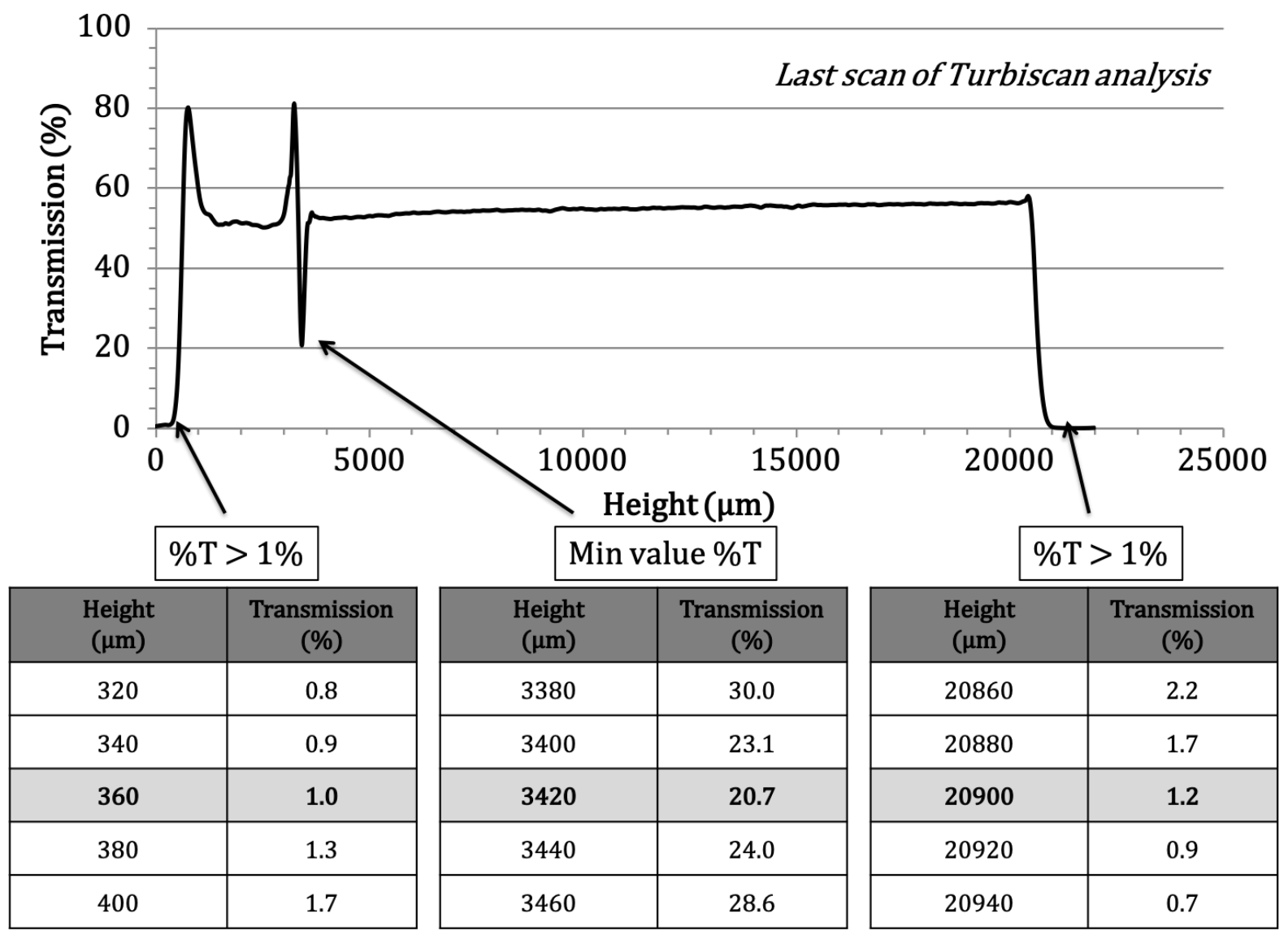

Figure S3. Method for determining height from Turbiscan transmission profiles - example for the transmission profile obtained with polymers in powder form and purified PEO at a concentration of $3 \mathrm{wt} \%$ 